Review

\title{
Scientific Evidence in Homeopathy: A Dynamic Database
}

Francesco V. Marino *

Federazione Italiana Associazioni Medici Omeopati, LOTUS Centro Medicina Olistica, Via Divisione Torino 600143 ROME, Italy; E-Mail:dr.fmarino@gmail.com

* Correspondence: Francesco V. Marino; E-Mail: dr.fmarino@gmail.com

Academic Editor: Bruno Galeazzi

Special Issue: $\underline{\text { Homeopathy }}$

OBM Integrative and Complementary Medicine 2021, volume 6 , issue 3

doi:10.21926/obm.icm.2103032
Received: April 27, 2021

Accepted: September 17, 2021

Published: September 28, 2021

\begin{abstract}
Homeopathy, a system of Traditional and Complementary Medicine (T\&CM, according to WHO definition), is under frequent attack by skeptics because of its supposed "lack of evidence." To overcome the distrust of skeptics and the public, many databases have been created, which focused on collecting all the published and indexed studies; however, none of these seem to be "comprehensive" and systematic enough. Therefore, this study aimed to evaluate the most reliable and available homeopathic studies in order to systematize the best evidence at all levels of research (studies based on humans, animals, and plants, randomized controlled trials [RCTs], observational studies, fundamental studies, etc.). A dynamic database was created containing 1,146 reliable pieces of evidence. It is being updated every three months and is available both for the whole homeopathic community and for the patients, Institutions, stakeholders, and skeptics. In the last 50 years, evidence in homeopathy has been increasing both at a quantitative and qualitative level. Unfortunately, it is not well known by the homeopaths themselves and also by the medical and scientific community. Therefore, a comprehensive database will not only support homeopaths but also assist in the better promotion and worldwide recognition of homeopathy as a significant field of medicine.
\end{abstract}

\section{Keywords}

Homeopathy; database; evidence; EBM; protocols; study design

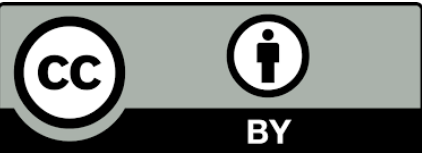

(C) 2021 by the author. This is an open access article distributed under the conditions of the Creative Commons by Attribution License, which permits unrestricted use, distribution, and reproduction in any medium or format, provided the original work is correctly cited. 


\section{Scientific Research in the Era of EBM}

Since the early 1990s, EBM (evidence-based medicine) has redefined the parameters for scientific research, especially in biomedicine and professional practice. In this context, the quality of clinical study has become crucial both in patient management and health policy choices. The quality of a clinical study must generally take account of ten aspects:

\section{Description of the characteristics of enrolled patients}

2. Study design

3. The sample size of patients

4. Description of the randomization

5. Blindness

6. Treatment description

7. Description of measurement modes

8. Patients who completed the study

9. Statistical suitability

10. Type of medical team

The study must also be published in a peer-reviewed journal, i.e., it must be assessed by a committee of referees. The journal must be indexed in biomedical databases (PubMed, Scopus, BVS, etc.), and it may have an Impact Factor (I.F.)

\section{Scientific Research in Homeopathic Medicine: Peculiarities and Criticalities}

One of the objections that has always been raised against homeopathy is the "lack of clinical evidence", which is the fruit of deficient or poor scientific research. Based on this, its therapeutic effect is credited to the placebo effect $[1,2]$. However, history indicates that homeopathy was developed under the sign of research and experimentation: Hahnemann was, in fact, the first physician to ever test and rigorously catalog the symptomatology produced by the pure action of substances on a healthy subject so that it could be used therapeutically on a patient. Following his teachings, long before the development of clinical trials, other homeopaths tested the actions of medicinal products with double-blind $[3,4]$ and multi-center study techniques. In terms of methodology, the leap in quality research in homeopathy dates to the 1970s-80s, when a series of studies with more stringent criteria started to be carried out. With the advent of the EBM culture (1991-92), scientific production has substantially increased both quantitatively and qualitatively, especially over the past decade. However, for correct evidence evaluation, it is necessary to take account of the peculiarities and criticalities central to homeopathy, such as:

- The personalization of the treatment and modulation of posology (the science of dosage) with respect to the practice of protocols usually adopted by "conventional" research, which is known to disregard individual differences.

- The dilutions used, where the remedies are often so diluted that they become undetectable in the pharmacokinetic analysis unless extremely sophisticated equipment is used. 


\section{A Dynamic Database: Rationale}

To verify the existence and value of scientific research in homeopathy, a "DYNAMIC DATABASE", which is a constantly updated database, was created (http://database.fiamo.org). Below are the inclusion/exclusion criteria considered for the creation of this database (Table 1).

Table 1 Inclusion/Exclusion Criteria.

Inclusion Criteria:

Published and indexed studies based on homeopathic medicinal products (since 1949) Both positive and negative studies References drawn from: Pubmed, Embase, SCOPUS, Core-Hom, Google Scholar, and BVS A relevant link to the abstract was included for each reference (sufficient and necessary condition)

\section{Exclusion Criteria:}

Studies based on complementary or alternative medicine (CAM) in general and/or mixed studies (with homeopathic medicinal products and CAM)

Books and conference proceedings Journals not accessible via the Internet

Posters

Educational magazines

Publications with editorial discretion

Publish and perish

Currently being reviewed (comments and brief notes)

Homeopathy-based research is being conducted in various fields; therefore, the database was organized accordingly based on the relevant areas of interest:

- Agro-homeopathy: It uses homeopathic medicinal products to treat plants, including plants grown in the greenhouse and open fields. It is based on standardized, quickly applicable, relatively inexpensive experiments, without ethical implication or placebo effect.

- Chemical-physical research: It studies the chemical-physical properties of extremely diluted solutions (EDS), where the diluted homeopathic medicine is dissolved into the solvent.

- Preclinical research (lab): It utilizes "in vitro" or "in vivo" models to study possible mechanisms of action of homeopathic medicinal products.

- Case reports: Individual clinical cases of patients treated with homeopathic medicinal products.

- Clinical research (observational or non-interventional studies): It explores the clinical effect of homeopathic medicinal products by observing the evolution of the disease/medical condition in response to the pharmacological therapy prescribed.

- Clinical research (RCT or interventional studies): It explores the clinical efficacy of homeopathic medicinal products compared with placebo or with a control drug.

- Qualitative or narrative systematic reviews: They are a collection of clinical studies, describing them with a qualitative and/or narrative approach.

- Systematic reviews with meta-analysis: They analyze the results of a series of clinical studies answering a specific clinical question, thereby statistically establishing the efficacy of the pharmacological therapy under study. 
- Veterinary: It assesses the clinical and experimental efficacy of homeopathic medicinal products in the treatment of farm and/or pet animals.

\section{Study Quality}

To assess the quality of the studies, evaluation scales are generally used where scores are collected and assigned to the parameters analyzed, such as study design, optimal conduct of the research under scrutiny, reporting quality, and completeness of the description of the study for publishing purposes (Table 2).

Table 2 Evaluation Scales.

\begin{tabular}{lll}
\hline STUDY DESIGN & QUALITY CHECKLIST & REPORTING CHECKLIST \\
GUIDELINES & GRADE & \\
$\begin{array}{l}\text { Systematic Review } \\
\text { Analysis }\end{array}$ & With & Meta- \\
Rct & Amstar & \\
$\begin{array}{l}\text { Controlled/Non-Randomized } \\
\text { Study }\end{array}$ & Jadad Scale & $\begin{array}{l}\text { Consort } \\
\text { Trend }\end{array}$ \\
Cohort Study & & \\
Case-Control Study & Newcastle-Ottawa Scale & Strobe \\
Longitudinal Study & Newcastle-Ottawa Scale & Strobe \\
\hline
\end{tabular}

Www.Equator-Network.Org

\section{Database: Instructions for Use}

All the studies included have been provided with "masks" to search for the main reference parameters of the study (Table 3 and Figure 1):

Table 3 Masks related to study parameters.

\begin{tabular}{llll}
\hline Year of publication & First author & Journal & Page/Volume \\
Title & Type & Keywords & Link to abstract/full text \\
\hline
\end{tabular}

For RCTs, other masks were added, referring to:

- Pathological condition studied

- Therapy with homeopathic medicinal products, individualized or not

- Published in a peer-reviewed journal, or not

- Comparison with placebo or OTP (Other Than Placebo) 


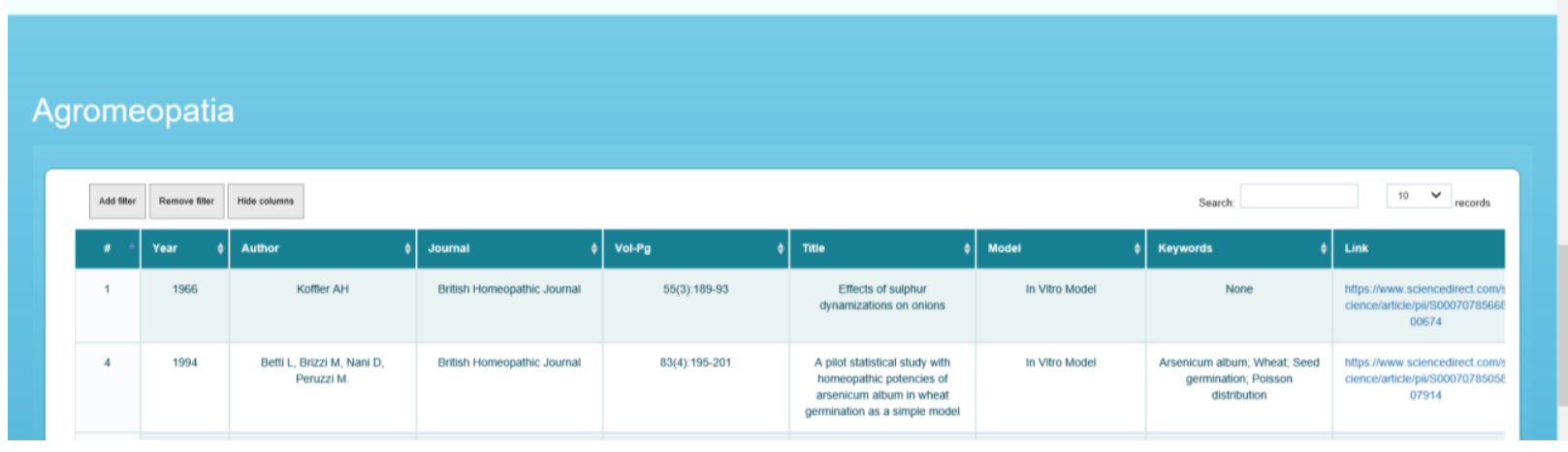

Figure 1 Masks related to study parameters.

Filters were added (Add filter) to make it easier to launch "historical" queries (e.g., Year, Author, Journal, Design, and Keywords) (Figure 2).

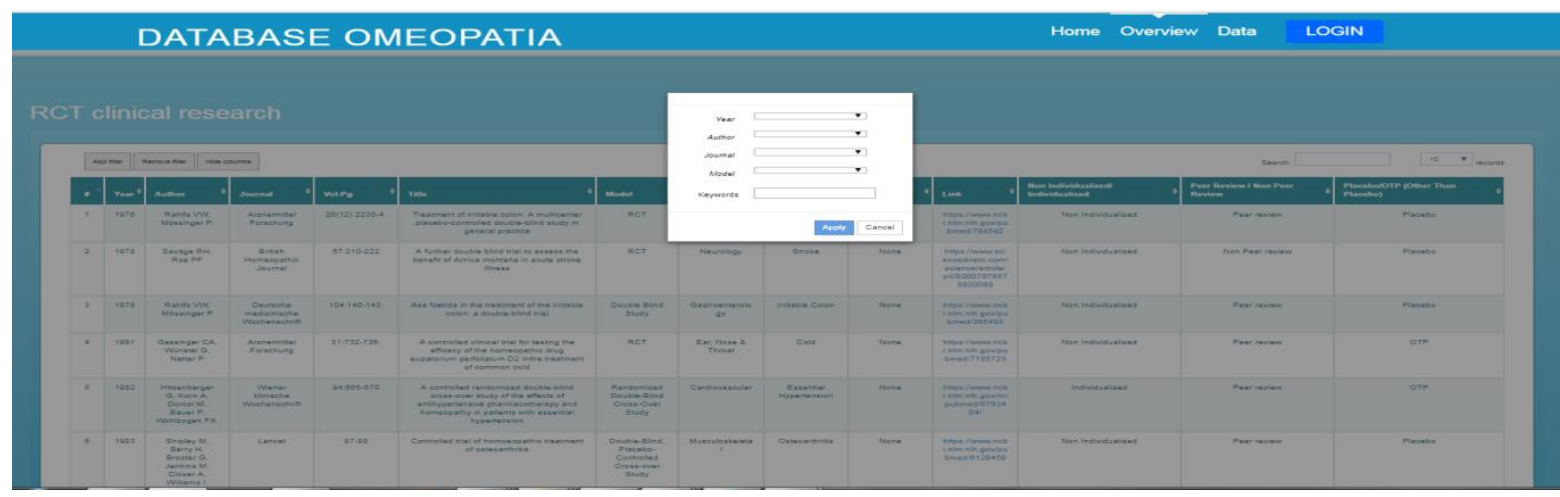

Figure 2 Filters: how to launch queries.

You can also sort by:

- Medicinal product, in the different sections, by clicking on the top right box (Search), e.g., Mercurius (Figure 3).

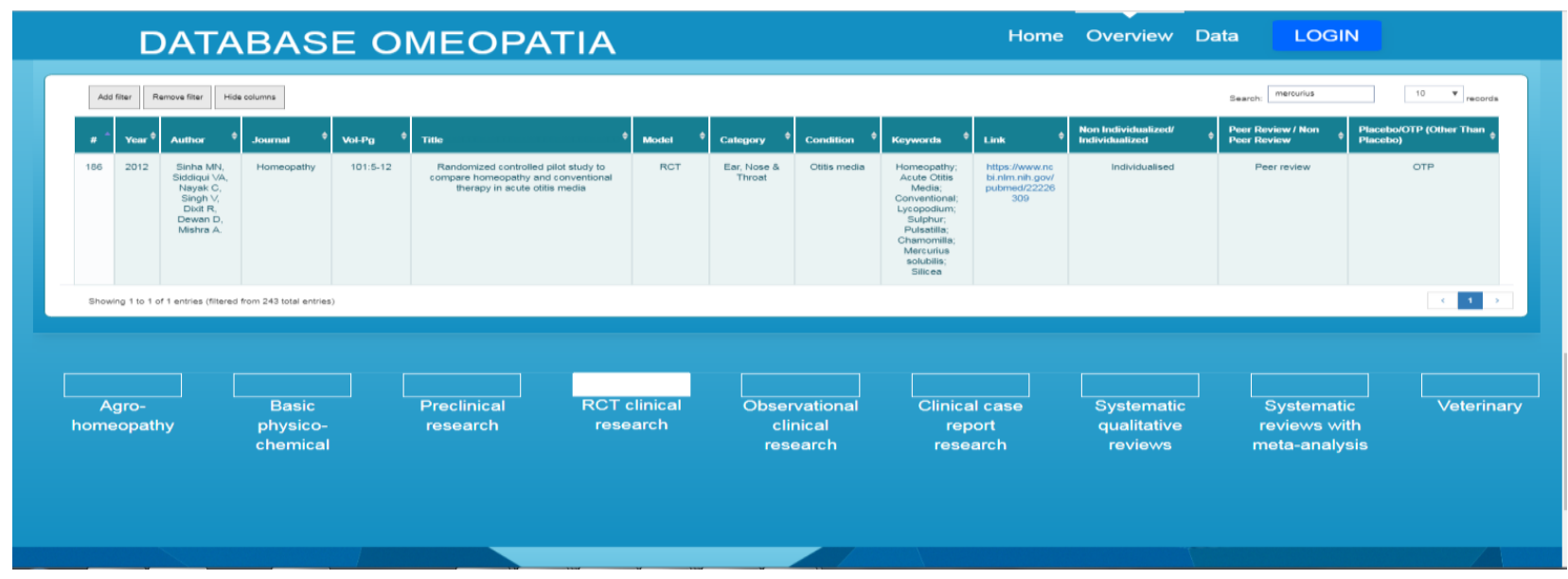

Figure 3 Filters: how to find medicines used in the studies. 
- Category (specialization), study design, pathological condition, etc., by clicking on the relevant column (Figure 4).

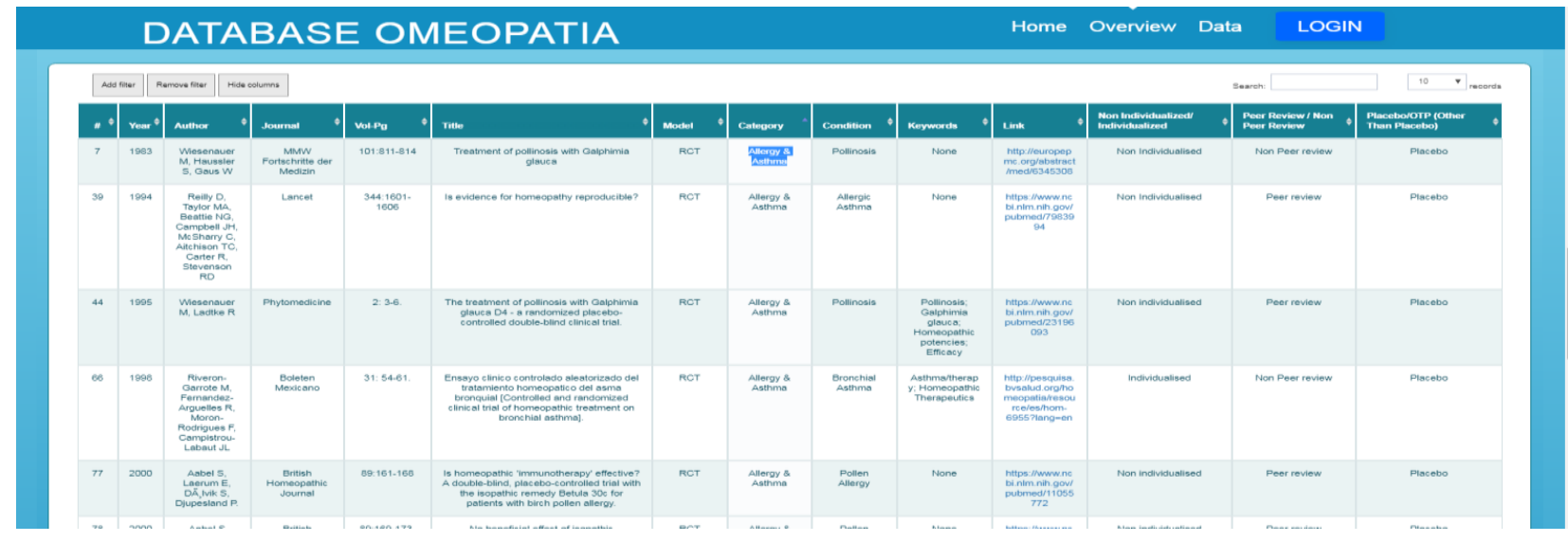

Figure 4 Filters: how to search for studies based on specialization, design, disease, etc.

\section{Results}

To facilitate the understanding of homeopathy, we created a database containing almost 1,146 reliable studies. The results of the below-mentioned studies indicate encouraging outcomes of homeopathic research:

$\begin{array}{ll}\text { Systematic reviews: } & 101 \\ \text { Metanalyses: } & 26 \\ \text { RCTs: } & 247 \\ \text { Observational: } & 128 \\ \text { Veterinary: } & 109 \\ \text { Agro-homeopathy: } & 96 \\ \text { Pre-clinical: } & 219 \\ \text { Chemical-physical: } & 156 \\ \text { Case reports: } & 64\end{array}$

Based on the above-mentioned special filters, it is possible to select the studies according to year, author, journal, title, topic, page, condition, model, and category, thereby making the search userfriendly.

The database, which is safe because of password protection, has been implemented in the Italian Federation of Homeopathic Associations and Homeopaths (FIAMO) website (https://database.fiamo.org/). Moreover, it is being updated every three months and shared with the whole (national and international) homeopathic community. It will also be available to patients, Institutions, stakeholders, and media.

From 2020, it became an official document recognized by the most important and qualified homeopathic organizations worldwide (LMHI, ECH, and EUROCAM). 


\section{Conclusions}

In the last 50 years, clinical studies on homeopathic medicines have been increasing both at a quantitative and qualitative level. Unfortunately, they are not well known by the homeopaths and also by the medical and scientific community. A comprehensive database is fundamental for the better outreach of easy-to-find high-quality research to all members of society, including the homeopathic community, scientists interested in the subject, and students.

\section{Author Contributions}

The author did all the research work of this study.

\section{Competing Interests}

The author has declared that no competing interests exist.

\section{References}

1. Linde K, Clausius N, Ramirez G, Melchart D, Eitel F, Hedges LV, et al. Are the clinical effects of homoeopathy placebo effects? A meta-analysis of placebo-controlled trials. Lancet. 1997; 350: 834-843.

2. Ernst E. Is homoeopathy a placebo? Br J Clin Pharmacol. 1990; 30: 173-174.

3. Jacobs J, Gloyd SS, Gale JL, Jiménez LM, Crothers D. Treatment of acute childhood diarrhea with homeopathic medicine: A randomized clinical trial in Nicaragua. Pediatrics. 1994; 93: 719-725.

4. Sorrentino L, Piraneo S, Riggio E, Basilicò S, Sartani A, Bossi D, et al. Is there a role for homeopathy in breast cancer surgery? A first randomized clinical trial on treatment with Arnica montana to reduce post-operative seroma and bleeding in patients undergoing total mastectomy. J Intercult Ethnopharmacol. 2017; 6: 1-8.

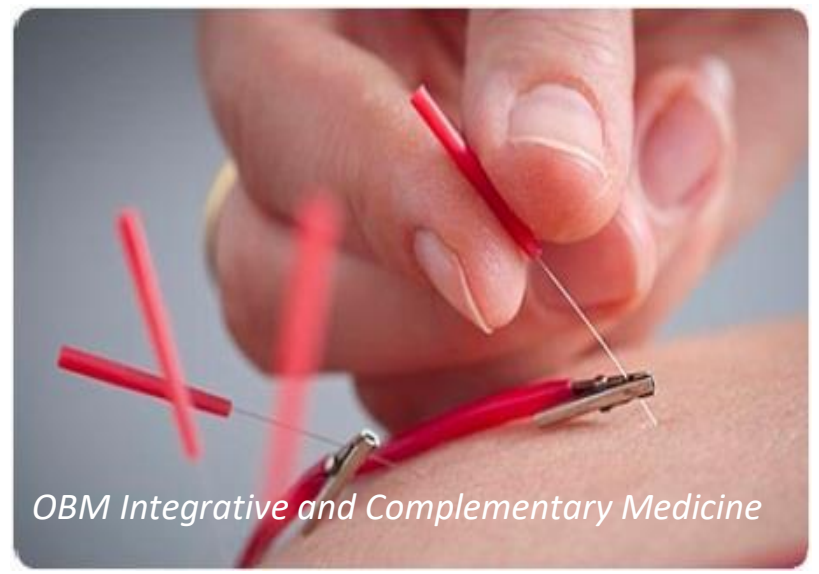

Enjoy OBM Integrative and Complementary Medicine by:

1. Submitting a manuscript

2. Joining in volunteer reviewer bank

3. Joining Editorial Board

4. Guest editing a special issue

For more details, please visit: http://www.lidsen.com/journals/icm 